\title{
Ambulatory recorded ST segment depression on ECG is associated with lower cognitive function in healthy elderly men
}

This article was published in the following Dove Press journal:

International Journal of General Medicine

13 August 2009

Number of times this article has been viewed

\section{Sölve Elmståhl \\ Linda Furuäng}

Department of Health Sciences, Division of Geriatric Medicine, Lund University, Malmö University Hospital, Malmö, Sweden

Correspondence: Sölve Elmståhl

Department of Health Sciences, Division of Geriatric Medicine, Lund University,

Malmö University Hospital, CRC,

SE-205 02 Malmö, Sweden

Tel +46 4039 I 320

Fax +464039 I3 I3

Email solve.elmstahl@med.lu.se
Abstract: ST segment depression (STDE) has been found to be associated with cardiovascular disease in the elderly. Studies of the relation of ambulatory STDE to cognitive function in elderly persons aged 80 years or above is lacking.

Objective: To study the association between STDE and cognition.

Design and participants: A cross-sectional cohort study of 88 81-year-old men from the population study "Men born in 1914" investigated in an outpatient research clinic. Measurements included ambulatory 24-hour electrocardiogram (ECG) monitoring and a cognitive test battery of six tests. Proportion of lower cognitive function was calculated for each test in relation to STDE during the day and at night-time.

Results: Fifty-eight percent of the men had STDE and a higher proportion with low visuospatial cognitive function was found among those with STDE compared to the others (84\% vs 59\%; $\mathrm{p}=0.014$ ). A significant trend was noted for subjects without STDE compared to STDE night-time less than 60 minutes and night-time more than 60 minutes for spatial and verbal cognitive functions $(p=0.022)$. No trends were noted for STDE daytime. Maximal STDE during night showed similar association to spatial function (Benton Visual Retention test, $r=-0.26$; $\mathrm{p}=0.028$ ). Even when seven subjects with a history of stroke were excluded, the occurrence of STDE was associated to lower visuospatial cognitive function compared to those without STDE ( $87 \%$ vs $57 \%$; $\mathrm{p}=0.004)$.

Conclusion: ST segment depression on ECG is common among elderly men and might be a vascular risk factor for cognitive deterioration.

Keywords: aged 80 and over, ST segment depression, ambulatory long-term ECG, cognition, cohort study, risk factors

\section{Introduction}

Previous studies have reported on occurrence of ST segment depression (STDE) during long-term electrocardiogram (ECG) recording (LTER) in relation to cardiovascular disease, risk factors such as hypertension and mortality. ${ }^{1-3}$ Silent myocardial ischemia is a common manifestation of coronary artery disease (CAD) and as much as $40 \%$ of elderly patients without a history of CAD may have this condition. ${ }^{4} \mathrm{CAD}$ is also a recognized risk factor for symptomatic ischemic stroke, but few studies have addressed the influence on asymptomatic stroke. Silent brain infarctions have been detected in about $30 \%$ in previous studies of patients with CAD and the clinical impact of this high number of clinically silent ischemic brain lesions is debated. ${ }^{5,6}$ However, there is support for that the cumulative burden of these lesions causes neuropsychological deficits and potentially increases the risk for vascular dementia. ${ }^{7}$ 
A previous population study, "Men born in 1914" of 68-year-old men reported that STDE during LTER was an independent risk factor of cardiac event in men without a history of ischemic heart disease (IHD) although STDE is recognized as a marker of IHD. ${ }^{3}$ The same cohort of men has now been followed and re-examined at the age of 81 years and a 24-hour LTER and a cognitive test battery were employed in a further step to examine the relationship between cardiovascular risk factors, especially STDE as marker of coronary heart disease, and cognitive functioning in the very elderly. To our knowledge, there has been no such study as yet on this topic concerned with subjects of this age or higher.

\section{Methods}

\section{Subjects}

In 1995-1996, 186 of the 266 surviving participants from the ongoing prospective population study on cardiovascular disease, "Men born in 1914", ,9 still living in Malmö agreed to take part in a new investigation. One man was excluded after failing to participate in the physical health examination. LTER was performed on 99 subjects of whom 88 had recordings of sufficient quality and also had completed a cognitive test battery. Approximately one hour was needed to complete the battery. In the previous examinations, 703 out of 809 men (87\%) participated in the first examination 1968 to 1969 , after an invitation of all men born even months the year 1914 in the city of Malmö and 500 men participated in the second examination in 1982 to 1983.

\section{Health examination}

LTER and cognitive testing were carried out for each of the subjects at the age of 81 . Information on stroke, likewise at the age of 81 , defined according to the ICD-9 classification $430.00-438.99,{ }^{10}$ was retrieved from the Stroke Registry database at the hospital. Information on background factors was taken from the previous examination at the age of 68 , in view of the extended time lag for any impact they might have on cognition.

Office blood pressure (BP) was measured with duplicate recordings using a manual sphygmomanometer with the subject in a sitting position. Hypertension was defined as an office systolic blood pressure (SBP) $\geq 160 \mathrm{mmHg}$, diastolic blood pressure (DBP) $>90 \mathrm{mmHg}$ or medication for hypertension. ${ }^{11}$ Thirty-three out of 88 (38\%) men had hypertension. Diabetes mellitus was defined as a fasting blood glucose level $\geq 7 \mathrm{mmol} / 1$ or taking medications for diabetes mellitus. ${ }^{12}$ Hyperlipidemia was defined as fasting triglycerides $\geq 2.3 \mathrm{mmol} / 1$ or cholesterol $\geq 6.5 \mathrm{mmol} / 1 .{ }^{13,14}$ Obesity was defined as body mass index (BMI) $>28$.

Ischemic heart disease was defined as the person's having a history of myocardial infarction or fulfilling of the diagnostic criteria of angina pectoris according to the Rose Questionnaire. ${ }^{15}$ Dementia was classified according to DSM-IV. ${ }^{16}$

Current smokers and ex-smokers were defined as smokers if they were consuming or had consumed at least $1 \mathrm{~g}$ of tobacco/day or had been smoking regularly for at least a year. Average alcohol consumption above $250 \mathrm{~g}$ weekly was defined as high. ${ }^{17}$ Marital status was categorized as unmarried/noncohabitant or married/cohabitant. Level of education was classified as secondary school or more with 10 years or more. Social class was determined on the basis of earlier occupation, working tasks and position, comprising blue-collar workers, low and medium-level white-collar workers, and persons in managerial positions, professionals with university degrees, and owners of business enterprises with employees. ${ }^{18}$

The ultrasound examination of the carotid artery was performed with an Acuson XP 10 Computed sonography system (Acuson, Mountain View, CA, USA), using a $7 \mathrm{MHz}$ B-mode real-time linear scanner including a $5 \mathrm{MHz}$ pulsed and color-coded Doppler and the method is described elsewhere. ${ }^{19}$ Carotid artery disease was defined as a maximum frequency shift (MFS) $>3,1 \mathrm{kHz}$ (corresponding to a stenosis of more than $30 \%$ of the cross-sectional diameter of the internal carotid artery lumen) or occlusion of carotid artery unilaterally or bilaterally. ${ }^{19}$ Ankle-brachial BP index recording of SBP was measured with a sphygmomanometer in both upper arms and at both ankle levels, using a mercury strain gauge as pulse detector. ${ }^{19}$ For each leg, an anklebrachial pressure index (ABPI) was calculated by dividing the ankle SBP with the highest upper arm SBP value.

\section{Long-term ECG recording}

LTER was monitored for 24 hours using a frequencymodulated ECG tape recorder (Micro AM-5600; Kontron Instruments, Bensheim, Germany), using two pairs of bipolar electrodes applied to the chest according to the ECG V2 and V6 locations and data was recorded on cassettes at a speed of $2 \mathrm{~mm} / \mathrm{s}$. The tape analysis for ST segment changes and cardiac arrhythmia were analyzed by one and the same technician using Oxford Medilog Analyzer (Oxford Instruments, Oxon, UK). The computerized tape analyses included a scanner and playback analyzer unit for the visual interpretation by the technician. 
Arrhythmia was detected by analyses of the R-R intervals. The ST segment was identified by two adjustable markers, one placed on the PR isoelectrical line, on the midpoint of the PR segment and the other one after the $J$ point at the end of the QRS complex on the ST segment between the $\mathrm{S}$ and $\mathrm{T}$ waves. No corrections were made for baseline STDE, in order to avoid any reduction of subjects with ischemia. The visual interpretation included the whole tape and parts of interest were printed out at $25 \mathrm{~mm} / \mathrm{sec}$ speed for analyses of STDE and arrhythmia in relation to heart rate in order to identify possible positional ST changes, especially during night. ST segment changes was defined as $>0.1 \mathrm{mV}$ $(1 \mathrm{~mm})$ planar or down-sloping shift from the isoelectric baseline occurring at 60 milliseconds after the J point, with the midpoint of PR as a reference and with a duration of at least $\geq 1$ minute. ${ }^{20}$ The maximal STDE was defined as the maximum value registered by the recorder. There were no restrictions on physical activity other than those necessary to obtain accurate readings. Tachyarrhythmia was defined as $>100$ beats per minute, bradyarrhythmia $<50$ beats per minute and supraventricular tachycardia as $>10$ coupled supraventricular beats.

\section{Measures of cognitive functioning}

The subjects were all tested by the same clinical psychologist. The cognitive test battery consisted of the following: the Mini-Mental State Examination (MMSE); ${ }^{21}$ S Swedish version of Block Design, which tests visuospatial, organizational ability;22 synonyms, which tests general verbal ability; ${ }^{23}$ a Swedish version of the original Verbal Paired Associates of Wechsler, testing immediate verbal memory; ${ }^{22}$ Digit Symbol Substitution Test, testing performance speed, visual-motor coordination, concentration, sustained attention and cognitive flexibility;22 and the Benton Visual Retention Test, which tests immediate visual and spatial recall..$^{24}$

\section{Statistics}

Differences in background data between participants and excluded subjects were explored using the chi-squared test, or Fisher's exact test in the case of small expected frequencies, and the Mann-Whitney U-test in the case of ordered groups.

For each of the cognitive tests, a result at or below a cutoff point one standard deviation below the mean for each of the specific test was classified as representing lower cognitive function. This cutoff was chosen for the purpose of identifying any lower cognitive functioning and not primarily to identify cognitive impairment or early stages of dementia.
The chi-squared test was used to analyze difference in proportion of lower cognitive functions in relation to occurrence of STDE during 24 hours as well as divided in three groups, no STDE, less than 60 minutes and more than 60 minutes, daytime and night-time $10.00 \mathrm{pm}$ to $06.00 \mathrm{am}$ ). Trend for linear by linear association between the three groups are given separately. Analyses were also made after exclusion of men with a history of myocardial infarction. None of the background factors had any significant association to the five different cognitive tests using chi-squared test, or Fisher's exact test, which is why no further statistical regression modeling was done. The criterion for inclusion of variables in the model was a 5\% significance level.

\section{Ethics}

The study was approved by the local ethics committee at Lund University. All subjects gave their informed consent.

\section{Results}

Background data for the participants and the excluded subjects are presented in Table 1. No differences were found between the study participants $(\mathrm{n}=88)$ and the excluded subjects ( $n=97$ ) on any of the retrieved background data, including history of hypertension, diabetes mellitus, hyperlipidemia, obesity, smoking, alcohol intake, physical activity, civil status, education, social class, known cardiovascular disease

Table I Background factors found for the group that was studied and for the subjects that were excluded

\begin{tabular}{llll}
\hline Background factor & $\begin{array}{l}\text { Study } \\
\text { population } \\
(\mathbf{n = 8 8 )}\end{array}$ & $\begin{array}{l}\text { Excluded } \\
(\mathbf{n}=97)\end{array}$ & p-value \\
\hline Hypertension & $49(56 \%)$ & $54(55 \%)$ & 0.843 \\
Diabetes mellitus & $\mathrm{I}(1 \%)$ & $4(4 \%)$ & $0.374^{*}$ \\
Hyperlipidemia & $29(3 \%)$ & $34(35 \%)$ & 0.845 \\
Obesity & $18(20 \%)$ & $12(12 \%)$ & 0.120 \\
Smoking, current & $26(30 \%)$ & $29(30 \%)$ & 0.999 \\
Myocardial infarction & $15(17 \%)$ & $6(15 \%)$ & 0.372 \\
Angina pectoris & $5(6 \%)$ & $5(5 \%)$ & $0.845 *$ \\
High alcohol & $4(5 \%)$ & $6(6 \%)$ & $0.75 I^{*}$ \\
consumption & & $83(85 \%)$ & 0.383 \\
Married/cohabitant & $78(89 \%)$ & $20(21 \%)$ & 0.360 \\
Secondary school & $15(17 \%)$ & & \\
or more & & $18(18 \%)$ & 0.912 \\
Social class & & $37(38 \%)$ & 0.912 \\
\hline $\begin{array}{l}\text { Blue collar workers } \\
\text { White collar workers }\end{array}$ & $14(16 \%)$ & $33(38 \%)$ &
\end{tabular}

Notes: Statistical analyses were performed using the chi-squared test, unless otherwise stated. *Statistical analysis using Fisher's exact test. 
and stroke. Mean values for the cognitive tests are presented in Table 2. Only one man had a MMSE score below 24.

Fifty-one of 88 men (58\%) had STDE during the 24-hour LTER. Twenty-two men had only STDE daytime and 29 men both daytime and night-time. The mean minutes and levels of STDE daytime were $294 \pm 367$ minutes and $2.7 \pm 1.4 \mathrm{~mm}$ $(\mathrm{n}=51)$. The corresponding values for STDE night-time $(\mathrm{n}=29)$ was $234 \pm 219$ minutes and $3.2 \pm 1.7 \mathrm{~mm}$ and only daytime $(\mathrm{n}=22)$ was $31 \pm 31$ minutes and $1.9 \pm 0.8 \mathrm{~mm}$. No subject had STDE only during night-time. Twenty-four men had a history of previous CAD with either myocardial infarction or angina until the age of 81 years and of the remaining 64 men without CAD still 56\% had STDE during the LTER indicating silent ischemia. Seventeen men with previous CAD had a history of angina pectoris.

In analyzing results relating to cognitive functioning, subjects with STDE had a higher proportion with low visuospatial cognitive function, defined by Block Design test, compared to the others ( $84 \%$ vs $59 \%$; $=0.014$ ) (Table 3 ).

A significant trend with lower spatial and verbal cognitive functions ( $p=0.022)$ was noted for subjects without STDE compared to STDE night-time less than 60 minutes and night-time more than 60 minutes. No trend was noted when STDE daytime was analyzed separately (data not shown). Correlation analyses of maximal STDE during night showed similar association to scores of spatial function (Benton Visual Retention test, $r=-0.26 ; \mathrm{p}=0.028$ ).

Seven men had a history of stroke and one of them dementia when they were examined at 81 years of age and data were analyzed after exclusion of these men. Similar association were noted between occurrence of STDE and higher proportion of low visuospatial function, assessed

Table 2 Results on the cognitive tests

\begin{tabular}{lllll}
\hline $\begin{array}{l}\text { Test } \\
\text { (maximum } \\
\text { points) }\end{array}$ & $\mathbf{n}$ & $\begin{array}{l}\text { Mean } \\
\text { (SD) }\end{array}$ & $\begin{array}{l}\text { Cutoff } \\
\text { point }\end{array}$ & $\begin{array}{l}\text { No. of subjects } \\
\text { with results at } \\
\text { or below the } \\
\text { cutoff point } \\
\mathbf{n}(\%)\end{array}$ \\
\hline $\begin{array}{l}\text { Block design (42 p) } \\
\text { Synonyms (30 p) }\end{array}$ & 85 & $14.1(5.2)$ & 9 & $18(19)$ \\
$\begin{array}{l}\text { Paired } \\
\text { associates (30 p) }\end{array}$ & 83 & $14.6(5.5)$ & 14 & $17(18)$ \\
$\begin{array}{l}\text { Digit symbol } \\
\text { substitution (90 p) }\end{array}$ & 79 & $28.9(10.1)$ & 20 & $17(10)$ \\
$\begin{array}{l}\text { Benton visual } \\
\text { retention (I0 p) }\end{array}$ & 71 & $4.5(1.4)$ & 3 & $21(26)$ \\
MMSE (30 p) & 87 & $28.3(1.7)$ & 27 & $23(24)$ \\
\hline
\end{tabular}

Abbreviations: SD, standard deviation; MMSE, Mini-Mental State Examination. by the Block Design test $(\mathrm{p}=0.004)$ and also a positive trend for higher proportion of low cognitive functioning, especially Block Design test, Benton Visual Retention, and Paired Associates, with more STDE night-time (Table 3). When men with a history of myocardial infarction were excluded, men with STDE had a higher proportion of lower visuospatial function (88\%) compared to men without STDE (Block Design test, 66\%; $\mathrm{p}=0.02$ ).

Atherosclerotic risk factors and cardiovascular morbidity expressed by a history of angina pectoris, myocardial infarction, ankle-arm index as an assessment of peripheral atherosclerosis and carotid stenosis for men with and without STDE are presented in Table 4. No differences between the groups were noted except for hyperlipidemia.

\section{Discussion}

Ambulatory STDE was associated to lower cognitive functions, both visuospatial and verbal functions in otherwise healthy elderly men. This was found for a 24 -hour period as well as for night-time periods separately and the associations remained after exclusion of men with a history of cerebrovascular disease.

The concept of lower cognitive function used in this study is not equivalent with low functioning, since the definition of one standard deviation could include subjects with normal functioning. Normal values for low cognitive functioning among elderly aged 80 years and above are not as common as for younger subjects, especially if they should be sampled from similar birth cohorts. The aim of this study was, however, to identify possible associations in the distribution of cognitive functioning and STDE in a group of elderly men that could be indicative of asymptomatic CAD. It cannot be excluded that subjects with less education or lower occupational status could perform below average in some of the verbal cognitive tests, thereby introducing an effect as confounders. ${ }^{25}$ However, the Benton Visual Retention test and the Block Design test assess visuospatial and organizational functioning and these tests were both changed in a similar pattern.

In a review by Vermeer and colleagues, silent brain infarctions are detected in $20 \%$ of healthy elderly by magnetic resonance imaging (MRI) ${ }^{26}$ The presence of these infarcts, without stroke-like symptoms, is related to a doubled risk of stroke and dementia and deficits of cognitive function. Only a longitudinal follow-up of the present study population will give an answer to this question.

No association was found for STDE daytime and it can only be speculated on whether the noted findings of STDE 
Table 3 ST segment depression (STDE) divided in three groups: no STDE, less than 60 minutes and more than 60 minutes night-time and proportion (\%) of subjects with lower cognitive functions. P values are given for comparisons between groups (Chi-squared) and for linear trend between the three groups not at all, $<60 \mathrm{~min}$ and $>60 \mathrm{~min}$, also after exclusion of subjects with cerebrovascular disease (CVD)

\begin{tabular}{|c|c|c|c|c|c|c|c|c|c|c|c|}
\hline $\begin{array}{l}\text { ST segment } \\
\text { depression (ST) }\end{array}$ & No & $\begin{array}{l}\text { Low } \\
\text { block } \\
\text { design }\end{array}$ & $\begin{array}{l}p \\
p \text { for } \\
\text { trend" }\end{array}$ & $\begin{array}{l}\text { Low } \\
\text { synonyms }\end{array}$ & $\begin{array}{l}\overline{p \text { for }} \\
\text { trend" }\end{array}$ & $\begin{array}{l}\text { Low } \\
\text { paired } \\
\text { associates }\end{array}$ & $\begin{array}{l}p \\
p \text { for } \\
\text { trend" }\end{array}$ & $\begin{array}{l}\text { Low digit } \\
\text { symbol } \\
\text { substitution }\end{array}$ & $\begin{array}{l}p \\
p \text { for } \\
\text { trend" }\end{array}$ & $\begin{array}{l}\text { Low Benton } \\
\text { Visual } \\
\text { Retention }\end{array}$ & $\begin{array}{l}p \\
p \text { for } \\
\text { trend" }\end{array}$ \\
\hline \multicolumn{12}{|l|}{ During 24 hours } \\
\hline no ST & 37 & $59 \%$ & 0.014 & $67 \%$ & 0.775 & $68 \%$ & 0.887 & $68 \%$ & 1.000 & $54 \%$ & 0.285 \\
\hline yes ST & 51 & $84 \%$ & & $78 \%$ & & $69 \%$ & & $71 \%$ & & $63 \%$ & \\
\hline \multicolumn{12}{|l|}{ Night-time } \\
\hline no ST & 59 & $71 \%$ & 0.209 & $79 \%$ & 0.701 & $63 \%$ & 0.089 & $92 \%$ & 0.335 & $65 \%$ & 0.059 \\
\hline$<60 \min \mathrm{ST}$ & 12 & $83 \%$ & $0.079 \pi$ & $83 \%$ & $0.692^{\pi}$ & $92 \%$ & $0.045^{\pi}$ & $91 \%$ & $0.404^{\pi}$ & $91 \%$ & $0.022^{\pi}$ \\
\hline$>60 \min \mathrm{ST}$ & 17 & $88 \%$ & & $71 \%$ & & $82 \%$ & & $94 \%$ & & $92 \%$ & \\
\hline \multicolumn{12}{|l|}{ CVD excluded } \\
\hline \multicolumn{12}{|l|}{ During 24 hours } \\
\hline no ST & 35 & $57 \%$ & 0.004 & $66 \%$ & 0.679 & $66 \%$ & 0.884 & $63 \%$ & 0.739 & $51 \%$ & 0.286 \\
\hline yes ST & 46 & $87 \%$ & & $78 \%$ & & $72 \%$ & & $72 \%$ & & $63 \%$ & \\
\hline \multicolumn{12}{|l|}{ Night-time } \\
\hline no ST & 55 & $68 \%$ & 0.124 & $76 \%$ & 0.879 & $60 \%$ & 0.054 & $71 \%$ & $0.36 \mathrm{I}$ & $61 \%$ & 0.044 \\
\hline$<60 \min \mathrm{ST}$ & 11 & $82 \%$ & $0.044 \pi$ & $82 \%$ & $0.942^{\pi}$ & $91 \%$ & $0.023 \pi$ & $91 \%$ & $0.302^{\pi}$ & $91 \%$ & $0.016 \pi$ \\
\hline$>60 \min \mathrm{ST}$ & 15 & $93 \%$ & & $73 \%$ & & $87 \%$ & & $79 \%$ & & $91 \%$ & \\
\hline
\end{tabular}

night-time reflect an underlying asymptomatic CAD or the severity of STDE. The latter one could be supported by the noted dose response association between higher proportion of lower cognitive functioning and more STDE at night-time indicating that men with night-time STDE has a more advanced IHD and or silent ischemia. Ambulatory 24-hour monitoring could possibly identify STDE as a risk factor for cognitive functioning. In a previous study the circadian variation of ischemic STDE was studied in patients with intermittent claudication and both SBP and DBP were increased during the STDE episodes. ${ }^{27}$ It is possible that the mechanism behind the cognitive findings in this study, often related to small vessel disease, is related to hypertensive episodes rather than silent ischemia per se. Another interpretation of this cross-sectional study is to co-occurrence of both cerebral degenerative disease and CVD since both are strongly related and share the same vascular risk factors. Earlier studies have reported that angina and myocardial infarction contribute to ischemic stroke, ${ }^{28}$ but in this study the association between silent STDE and cognition remained when men with a history of cardiac events were excluded as well as after exclusion of men with stroke. It is known that a high proportion of individuals older than 65 years, almost a fourth, have asymptomatic coronary artery stenosis at high risk for future coronary events. ${ }^{29}$ Whether STDE can be used to identify also cerebral events remains to be studied. The results that the higher degree of night-time STDE was associated to lower cognitive impairment indicate a possible dose response association. The lack of association among men with only STDE daytime might be explained by a less severe STDE in this group compared to men with night-time, both less time of STDE, 31 minutes versus 234 minutes and less degree of segment depression STDE (1.9 $\mathrm{mm}$ vs $3.2 \mathrm{~mm}$ ).

A limitation of the study is that there are only male subjects. None of the background data for the excluded subjects diverged from that for the participants included in the study. The fact that only one subject was diagnosed as having dementia could imply a selection of cognitively more intact persons, also perhaps more willing and capable of participating in such a study, and possibly healthier than average for their age, although in other respects the men invited to participate appear to have been representative for men of their age in the community. Basically the results of the study appear to be reasonably valid for an elderly population of men without the diagnosis of dementia. No associations were found between the different cognitive tests and possible confounders to cognition like education, social class, lifestyle factors and diseases related to cognitive deterioration like stroke. Furthermore, most important was 
Table 4 Comparison of atherosclerotic risk factors and cardiovascular morbidity between men with and without ST segment depression (STDE)

\begin{tabular}{llll}
\hline Background factor & $\begin{array}{l}\text { STDE yes } \\
(\mathbf{n}=\mathbf{5} \text { I) }\end{array}$ & $\begin{array}{l}\text { STDE no } \\
(\mathbf{n}=\mathbf{3 7})\end{array}$ & p-value \\
\hline Hypertension & $29(56 \%)$ & $20(55 \%)$ & $\mathrm{ns}$ \\
Diabetes mellitus* & $0(0 \%)$ & $1(3 \%)$ & $\mathrm{ns}$ \\
Hyperlipidemia & $21(41 \%)$ & $8(22 \%)$ & 0.04 \\
Obesity & $10(20 \%)$ & $8(22 \%)$ & $\mathrm{ns}$ \\
Smoking, current & $12(24 \%)$ & $14(38 \%)$ & $\mathrm{ns}$ \\
High alcohol consumption* & $1(2 \%)$ & $3(8 \%)$ & $\mathrm{ns}$ \\
Myocardial infarction & $11(22 \%)$ & $6(17 \%)$ & $\mathrm{ns}$ \\
Angina pectoris & $11(22 \%)$ & $6(17 \%)$ & $\mathrm{ns}$ \\
Carotid stenosis" & $8(16 \%)$ & $5(14 \%)$ & $\mathrm{ns}$ \\
Ankle-arm index" & $1.04 \pm 0.16$ & $1.04 \pm 0.19$ & $\mathrm{~ns}$ \\
Blood pressure mmHg & & & \\
$\quad$ SBP & $145 \pm 17$ & $142 \pm 12$ & $\mathrm{~ns}$ \\
$\quad$ DBP & $82 \pm 6$ & $80 \pm 6$ & $\mathrm{~ns}$ \\
ECG & & & \\
Tachyarrhythmia $>100 / m i n$ & $64.7 \%$ & $64.9 \%$ & $\mathrm{~ns}$ \\
Bradyarrhythmia $<50 / m i n$ & $29.4 \%$ & $24.3 \%$ & $\mathrm{~ns}$ \\
Supraventricular & $39.2 \%$ & $45.9 \%$ & $\mathrm{~ns}$ \\
Tachyahrrythmia & & & \\
\hline
\end{tabular}

Notes: Statistical analyses were performed using the chi-squared test, unless otherwise stated; *Statistical analysis using Fisher's exact test; "missing data on three subjects. Abbreviations: DBP, diastolic blood pressure; ECG, electrocardiogram; SBP, systolic blood pressure.

that similar findings were noted after exclusion of men with cerebrovascular disease indicating that the findings were not confounded by the occurrence of CVD.

Another limitation of this study is the risk for false positive detection and the sensitivity of detected STDE by Holter ECG recordings is limited, and other techniques, scintigraphy and echocardiography are preferably better for studies of myocardial ischemia. ${ }^{30}$ A previous study by Bjerregaard and colleagues showed that detecting ischemic events $80 \mathrm{~ms}$ after the J-point instead of $60 \mathrm{~ms}$, used in this study, increased the number of ischemic events because most of these events are down-sloping..$^{20}$ The inclusion criteria of $0.1 \mathrm{mV}$ J-point depression will also reduce detected ischemic events. The consequences for this study could be that some potential ischemic events were undetected applying these criteria that might reduce statistical power.

However, strength of this study is the rather large sample of a general longitudinal population based cohort of elderly men investigated with a standardized protocol by one and the same physician, psychologist and laboratory technician. By the use of National Patient Registry information on medical history and in-patient diagnosis was also retrieved from medical records. No confounding was noted by other atherosclerotic risk factors or vascular morbidity in relation to the occurrence of STDE among the investigated men. Furthermore, there was no indication of selection bias between included and excluded men.

One implication of this study is to highlight the need of further longitudinal studies of STDE and risk for cognitive deterioration among the elderly, especially the incidence of mild cognitive impairment and vascular dementia. It could only be speculated whether common risk factor pattern explains the present findings and or if ambulatory LTER can be used in the vascular risk assessment of cognitive deterioration for elderly patients. Nevertheless, irrespectively if the finding is an expression of a more generalized atherosclerosis, the clinical relevance of identifying a possible risk group before they develop mild cognitive impairment and vascular dementia remains to be confirmed. The use of LTER is a noninvasive and easy tool to get important clinical information.

\section{Acknowledgments}

Financial support of the study was provided by the Swedish Research Council (K2004-27X-15016-01A) and the Faculty of Medicine at Lund University.

\section{References}

1. Stern S, Tzivoni D. early detection of silent ischaemic heart disease by 24-hour electro-cardiographic monitoring of active subjects. Br Heart J. 1974;36:481-486.

2. Schang SJ, Pepine CJ. Transient asymptomatic ST segment depression during daily activity. Am J Cardiol. 1977;39:396-402.

3. Hedblad B, Jull-Möller S, Svensson K, et al. Increased mortality in men with ST segment depression during $24 \mathrm{~h}$ ambulatory long-term ECG recording. Eur Heart J. 1989;10:149-158.

4. Deedwania PC. Silent myocardial ischaemia in the elderly. Drugs Aging. 2000;16:381-389.

5. Tanaka H, Sueyoshi K, Nishino M, et al. Silent brain infarction and coronary artery disease in Japanese patients. Arch Neurol. 1993;50: 706-709.

6. Modrego Pardo PJ, Labrador Furster T, Torres Nuez J. Silent brain infarctions in patients with coronary heart diseases. A Spanish population survey. J Neurol. 1998;245:93-97.

7. Bendszus M, Stoll G. Silent cerebral ischaemia: hidden fingerprints of invasive medical procedures. Lancet Neurol. 2006;5:470-471.

8. Reinprecht F, Elmståhl S, Janzon L, et al. Incidence and progression of carotid stenosis in elderly men: Thirteen-year follow-up of the population cohort "Men born in 1914". Int J Angiol. 2002;11: $132-138$.

9. Reinprecht F, Elmståhl S, Janzon L, et al. Hypertension and changes of cognitive function in 81-year old men. A 13-year follow-up of the population study "Men born in 1914", Sweden. J Hypertens. 2003;21:57-66.

10. World Health Organization. International Classification of Diseases, Injuries and Causes of Death. 9th revision (ICD-9). Geneva, Switzerland: World Health Organization; 1975. 
11. 1999 World Health Organization-International Society of Hypertension Guidelines for the Management of Hypertension. Guidelines Subcommittee. J Hypertens. 1999;17:151-183.

12. Carroll JJ, Smith N, Babson AL. A colorimetric serum glucose determination using hexokinase and glucose-6-phosphate dehydrogenase. Biochem Med. 1971;4:171-180.

13. Roeschlau P, Bernt E, Gruber W. Enzymatic determination of total cholesterol in serum. Z Klin Chem Klin Biochem. 1974;12:226.

14. Wahlefeld A. Triglycerides. Determination after enzymatic hydrolysis. In: Methods of Enzymatic Analysis. 2nd ed. New York, NY Academic Press; 1974. p. 1831.

15. Rose GA, Blackburn H. Cardiovascular survey methods. Monogr Ser World Health Organ. 1968;56:1-188.

16. American Psychiatric Association. Diagnostic and Statistical Manual of Mental Disorders: DSM-IV. 4th ed. Washington, DC: American Psychiatric Press Inc; 1994.

17. Royal College of Psychiatrists. Alcohol: our favourite drug. London, UK: Traviston; 1986.

18. Swedish National Central Bureau of Statistics. Swedish Socioeconomic Classification. Reports on statistical coordination: 4 [in Swedish]. Stockholm, Sweden: Swedish National Central Bureau of Statistics; 1982.

19. Reinprecht F, Elmståhl S, Janzon L, Andre-Petersson L. Hypertension and changes of cognitive function in 81-year old men. A 13-year follow-up of the population study "Men born in 1914", Sweden. J Hypertens. 2003;21:57-66.

20. Bjerregaard P, El-Shafei A, Kotar SL, et al. ST segment analysis by Holter monitoring. Ann Noninvasive Electrocardiol. 2003;8: 2000-2007.
21. Folstein MF, Folstein SE, McHugh PR. "Mini-mental state". A practical method for grading the cognitive state of patients for the clinician. J Psychiatr Res. 1975;12:189-198.

22. Wechsler D. Wechsler Adult Intelligence Scale. Revised manual. New York, NY: Psychological Corporation; 1981.

23. Dureman I, Sälde H. DS-batteriet. Manual [in Swedish]. Stockholm, Sweden: Psykologiförlaget; 1971.

24. Benton A. The Revised Visual Retention Test: clinical and experimental applications. 4th ed. New York, NY: Psychological Corporation; 1974.

25. Whittle C, Corrada MM, Dick M, et al. Neuropsychological data in nondemented oldest old: the 90+ Study. J Clin Exp Neuropsychol. 2007;29:290-299.

26. Vermeer SE, Longstreth WT, Koudstaal PJ. Silent brain infarcts: a systematic review. Lancet Neurol. 2007;6:611-619.

27. Svensson P, Niklasson U, Östergren J. Episodes of ST-segment depression is related to changes in ambulatory blood pressure and heart rate in intermittent claudication. J Intern Med. 2001;250: 398-405.

28. Wolf PA, Cobb JL, D'Agostino RB. Epidemiology of stroke. In: Barnett HJM, Mohr JP, Stein BM, Yatsu FM, editors. Stroke: pathophysiology, diagnosis and management. 2nd ed, vol I. New York, NY: Churchill Livingstone; 1992.

29. Fleg JL. Myocardial iscemia in the asymptomatic older patient. Am J Geriatr Cardiol. 1998;7:54-59.

30. Erne P, Schoenenberger A, Zuber M, et al. Effects of anti-ischaemic drug therapy in silent myocardial ischaemia type I: the Swiss Interventional study on silent ischaemia type I (SWISS I): a randomized, controlled pilot study. Eur Heart J. 2007;28:2110-2117.
International Journal of General Medicine

\section{Publish your work in this journal}

The International Journal of General Medicine is an international, peer-reviewed open-access journal that focuses on general and internal medicine, pathogenesis, epidemiology, diagnosis, monitoring and treatment protocols. The journal is characterized by the rapid reporting of reviews, original research and clinical studies across all disease areas.

\section{Dovepress}

A key focus is the elucidation of disease processes and management protocols resulting in improved outcomes for the patient.The manuscript management system is completely online and includes a very quick and fair peer-review system. Visit http://www.dovepress.com/ testimonials.php to read real quotes from published authors. 